\title{
Quasinormal modes of black holes in anti-de Sitter space: a numerical study of the eikonal limit
}

\author{
Jaqueline Morgan, ${ }^{1 *}$ Vitor Cardoso,,${ }^{2,3 \dagger}$ Alex S. Miranda, ${ }^{4 \ddagger}$ C. Molina, ${ }^{5 \S}$ and Vilson T. Zanchin ${ }^{1 \uparrow}$ \\ ${ }^{1}$ Centro de Ciências Naturais e Humanas, Universidade Federal do ABC, \\ Rua Santa Adélia 166, 09210-170 Santo André, SP, Brazil \\ ${ }^{2}$ Centro Multidisciplinar de Astrofísica - CENTRA, Dept. de Física, \\ Instituto Superior Técnico, Av. Rovisco Pais 1, 1049-001 Lisboa, Portugal \\ ${ }^{3}$ Department of Physics and Astronomy, The University of Mississippi, University, MS 38677-1848, USA \\ ${ }^{4}$ Instituto de Física, Universidade Federal do Rio de Janeiro, Caixa Postal 68528, RJ 21941-972, Brazil and \\ ${ }^{5}$ Escola de Artes, Ciências e Humanidades, Universidade de São Paulo, \\ Avenida Arlindo Bettio 1000, 03828-000 São Paulo, SP, Brazil
}

\begin{abstract}
Using series solutions and time-domain evolutions, we probe the eikonal limit of the gravitational and scalarfield quasinormal modes of large black holes and black branes in anti-de Sitter backgrounds. These results are particularly relevant for the AdS/CFT correspondence, since the eikonal regime is characterized by the existence of long-lived modes which (presumably) dominate the decay timescale of the perturbations. We confirm all the main qualitative features of these slowly-damped modes as predicted by Festuccia and Liu [14] for the scalarfield (tensor-type gravitational) fluctuations. However, quantitatively we find dimensional-dependent correction factors. We also investigate the dependence of the QNM frequencies on the horizon radius of the black hole (brane) and the angular momentum (wavenumber) of vector- and scalar-type gravitational perturbations.
\end{abstract}

PACS numbers: 04.70.-s, 11.25.Tq, 11.10.Wx, 04.50.-h,

\section{INTRODUCTION}

The characteristic modes of black holes and black branes are eigenmodes of these systems which convey important information about the background geometry. These systems are intrinsically dissipative (energy flows to the event horizon and/or towards the spatial infinity) and therefore their eigenmodes are not stationary. One calls the eigenmodes of these dissipative systems quasinormal modes (QNMs) [1, 2, 3, 4]. Within the anti-de Sitter/Conformal Field theory (AdS/CFT) duality [5, 6, 7], these modes serve as an important tool for determining the near-equilibrium properties of strongly coupled quantum field theories, in particular their transport coefficients such as viscosity, conductivity and diffusion constants [1, 8]. Recently, QNMs have also been used to study properties like the "meson melting" in D3/D7-brane models [9, 10, 11] and the spectrum of collective excitations of holographic superconductors [12, 13].

It is thus of interest to understand the spectrum of black holes and black branes in asymptotically anti-de Sitter (AdS) spacetimes. Recent studies by Festuccia and Liu (henceforth FL) have shed light on this issue, predicting the existence of long-lived modes in asymptotically AdS black hole (BH) geometries [14]. Their work shows that the eikonal limit in AdS depends sensitively on the relative size of the black hole. For small black holes, they find exponentially long-lived modes, which can be thought of as modes trapped inside the potential barrier. If we write $\omega=\omega_{R}-i \omega_{I}$ for the typical "energy"

\footnotetext{
*Electronic address: jaqueline.morgan@ufabc.edu.br

${ }^{\dagger}$ Electronic address: vitor.cardoso@ist.utl.pt

‡Electronic address: astmiranda@if.ufrj.br

$\S$ Electronic address: cmolina@usp.br

『Electronic address: zanchin@ufabc.edu.br
}

eigenvalue, these modes take the Bohr-Sommerfeld form

$$
2 i \int_{r_{b}}^{\infty} Q d r=\pi\left(2 n+\frac{5}{2}\right), \quad n=0,1, \ldots
$$

where

$$
Q \equiv \frac{1}{r f} \sqrt{(l+1 / 2)^{2} f-r^{2} \omega_{R}^{2}},
$$

$f$ is the black-hole horizon function (see Eq. (2.1) below), and $l$ is the angular momentum of the perturbation. Accordingly, their lifetime is dictated by a "tunneling probability" of the form $\omega_{I} \propto \exp \left(-2 \int_{r_{c}}^{r_{b}} Q d r\right)$, where $r_{b}$ and $r_{c}<r_{b}$ are two real zeros (turning points) of $Q$, and the proportionality coefficient is given in [15]. This prediction is supported by numerical studies for small AdS black holes [15].

On the other hand, the nature of the long-lived modes for large black holes is completely different, since no trapped modes are allowed in the large black-hole regime. A consequence of this fact is that the "Breit-Wigner resonance method" used to investigate small AdS black holes fails to give reliable results in the large black-hole regime [15]. These modes are also expected to be long-lived [14] (see Section $\amalg \mathrm{C}$ below). Thus these modes will presumably dominate the BH's response to arbitrary perturbations, hence the thermalization timescale in the dual CFT. Since their existence may be very relevant for the AdS/CFT conjecture, we decided to investigate numerically these long-lived modes. We use two, well established methods to study the QNMs of black holes. One is the series solution expansion [16, 17, 18], which outputs the characteristic frequencies directly and is especially well suited for large black holes. By using time-domain methods, in particular the scattering of Gaussian wavepackets [19, 20] (see also [21]), we confirm and expand the series solution results and furthermore show that these modes are excited in physically interesting situations. As a by-product, we confirm once more that there are no late-time tails in this geometry. 


\section{FORMULATION OF THE PROBLEM}

\section{A. The background spacetime}

BH's in asymptotically AdS spacetimes form a class of solutions which is interesting from a theoretical point of view and central for the study of strongly coupled field theories at finite temperature in the gauge/gravity duality framework. We are interested in a simple class of non-rotating, uncharged $d$-dimensional Schwarzschild-AdS black holes with line element

$$
d s^{2}=-f d t^{2}+f^{-1} d r^{2}+r^{2} d \Omega_{d-2}^{2},
$$

where $f(r)=1+r^{2} / R^{2}-r_{0}^{d-3} / r^{d-3}$ and $d \Omega_{d-2}^{2}$ is the metric of the unitary $(d-2)$-sphere. The AdS curvature radius $R$ is related to the cosmological constant $\Lambda$ by $R^{2}=$ $-(d-2)(d-1) / 2 \Lambda$. The parameter $r_{0}$ is proportional to the mass $M$ of the black hole: $M=(d-2) A_{d-2} r_{0}^{d-3} / 16 \pi$, where $A_{d-2}=2 \pi^{(d-1) / 2} / \Gamma[(d-1) / 2]$. The well-known Schwarzschild geometry corresponds to $R \rightarrow \infty$. The blackhole horizon radius $r=r_{+}$is the (unique) positive real root of $f(r)=0$.

In particular, we want to focus here on the large black hole regime, $r_{+} / R \rightarrow \infty$. In this case, the above geometry goes over to a $d$-dimensional plane-symmetric spacetime (black brane) [16], which is also an exact solution of Einstein's equations [22, 23, 24, 25]. The black brane has the following line element

$$
d s^{2}=-f(r) d t^{2}+r^{2} \sum_{i=1}^{d-2} d x^{i} d x_{i}+\frac{1}{f(r)} d r^{2}
$$

where $f(r)=r^{2} / R^{2}-r_{+}^{d-1} /\left(R^{2} r^{d-3}\right)$. The Hawking temperature of the black hole 2.1 is

$$
T=\frac{(d-1) r_{+}^{2}+(d-3) R^{2}}{4 \pi r_{+} R^{2}}
$$

and it reduces to $T=(d-1) r_{+} / 4 \pi R^{2}$ for large black holes, which is the Hawking temperature of the black brane (2.2).

\section{B. Gravitational perturbations}

Gravitational perturbations in these backgrounds were considered in Ref. [17, 26, 27] for $d=4$ and in [28, 29] for higher dimensions. In a generic number of dimensions, the gravitational perturbations can be divided in three different types, the tensor-, vector- and scalar-type perturbations. These can all be reduced to a master wave equation of the form

$$
f^{2} \frac{d^{2} \Psi}{d r^{2}}+f f^{\prime} \frac{d \Psi}{d r}+\left(\omega^{2}-V(r)\right) \Psi=0,
$$

where the potential $V(r)$ depends on both the type of perturbation and on basis functions used to separate the coordinates of the $(d-2)$-dimensional hypersurface of constant $r$ and $t$.
For instance, for tensor-type perturbations in the black-hole background 2.1,

$$
\frac{V}{f}=\frac{l(l+d-3)}{r^{2}}+\frac{(d-2)(d-4)}{4 r^{2}} f+\frac{(d-2) f^{\prime}}{2 r} .
$$

Here, the angular number $l$ is related to the eigenvalue of the hyper-spherical functions used to factor out the dependence on the $(d-2)$-spherical angles.

For the black brane background (2.2), the separation of trivial dimensions is achieved through the Ansatz $e^{i \vec{q} \cdot \vec{x}}$ and one ends up with the large $r_{+} / R$ limit of 2.5,

$$
\frac{V}{f}=\left[\frac{q^{2}}{r^{2}}+\frac{(d-2)(d-4)}{4 r^{2}} f+\frac{(d-2) f^{\prime}}{2 r}\right] .
$$

The explicit form of $V(r)$ for the vector- and scalar-type perturbations can be found in Refs. [17, 28, 29]. The potential for tensor-type gravitational perturbations is equal to the potential for scalar-field perturbations, so our results are also valid for spin-0 fields.

We will be interested in the large- $l, q$ limit of the QNM frequencies, i.e., characteristic $\omega$ 's for which the solutions to 2.4 satisfy the appropriate boundary conditions. Note that in this limit one can formally identify $q$ with $l$ in the black hole/black brane spacetimes. Hereafter we will always refer to $q$, with the understanding that the replacement $q \rightarrow l$ describes large black holes.

An important characteristic of classical field evolutions on asymptotically AdS spacetimes is the variety of choices for the boundary conditions at spatial infinity. In general, these can be Dirichlet, Neumann or Robin boundary conditions. So we need to establish an objective criterion before to choose a specific condition. In the AdS/CFT context, a natural criterion is such that the QNM frequencies of a certain field correspond to poles of two-point correlation functions of the dual operator in the boundary field theory [1, 30, 31, 32].

When we consider a variable $\Psi$ such that the master equation for the gravitational perturbations is of the form (2.4), in general the Dirichlet condition at spatial infinity is the 'correct' boundary condition. There is only one exception: for scalar-type perturbations in four spacetime dimensions, the boundary condition that leads to QNM frequencies corresponding to poles of retarded correlation functions is of Robin type (see Refs. [33, 34] for a discussion in $d=4,5$ and [36] for an arbitrary number of dimensions.)

\section{Long-lived modes in the eikonal limit: analytical prediction}

In asymptotically AdS spacetimes the eikonal limit is especially interesting, since large- $q$ modes can be very long-lived [14, 16]. A WKB analysis suggests that for the tensor-type gravitational perturbations (and therefore also scalar fields) 
and $r_{+} / R \gg 1$ [14], the following asymptotic behavior holds

$$
\begin{aligned}
R \omega^{\mathrm{FL}} & =q+\Pi_{n}\left(\frac{r_{+}}{R}\right)^{\frac{2 d-2}{d+1}} q^{-\frac{d-3}{d+1}}, \\
\Pi_{n} & \equiv\left(\sqrt{\pi}\left[\frac{d+1}{2}+2 n\right] \frac{\Gamma\left(\frac{3 d-1}{2 d-2}\right)}{\Gamma\left(\frac{1}{d-1}\right)}\right)^{\frac{2 d-2}{d+1}} e^{-\frac{22 \pi}{d+1}},
\end{aligned}
$$

as $q \rightarrow \infty$. So large- $q$ modes are very long-lived, and they could play a prominent role in the BH's response to generic perturbations. This is at variance with the asymptotically flat case, where the damping timescale is roughly constant as $q$ varies. Notice also that the scaling with the $\mathrm{BH}$ size differs from that of the weakly- and highly-damped modes.

\section{QUASINORMAL FREQUENCIES}

\section{A. Methods}

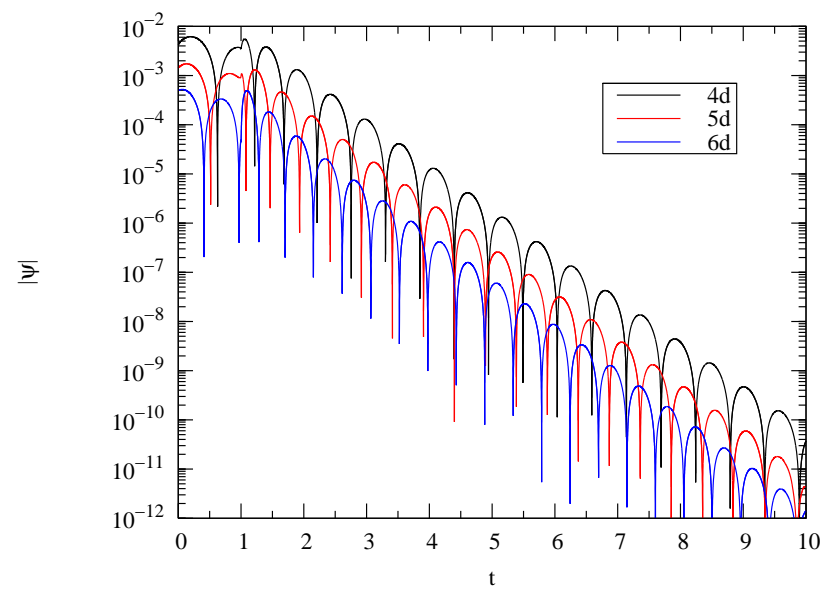

FIG. 1: Typical time-domain evolution of a gaussian wavepacket resulting in ringdown (top to bottom $d=4,5,6$ ). Here we take $\psi(u,-30)=0, \psi(0, v)=\exp \left[-(v-25)^{2} / 18\right]$ and $q=5$, where $u, v$ are standard null coordinates [19, 20]. The signal is measured at $r_{*} / R=-1$, where $d r / d r_{*}=f(r)$ and spatial infinity is at $r_{*}=0$.

We use two conceptually different methods to determine the gravitational QNM frequencies of the spacetimes (2.1) and [2.2), and both methods yield consistent results, within the expected error bars. The first consists on a series expansion method [1, 16], which reduces the problem to finding roots of a polynomial. This method is well suited to large black holes and black branes, though the convergence properties worsen for large wavenumber $q$. In fact, for larger dimensions $(d>6)$ and higher overtones $(n>1$ or 2$)$ the problems of convergence of the series solution arise even for intermediate wavenumber values $\left(R q / r_{+} \sim 10\right)$.

The second method employed in this work consists on a direct time-evolution in these backgrounds [19, 20]. A particular example is shown in Figure 1, which shows the timedevelopment of a Gaussian wavepacket in a $r_{+} / R=1$ black brane. The ringdown is characterized by the decay timescale and ringing frequency, which can directly be extracted from the slope and frequency of the signal above. Equivalently, we characterize the QNMs by a complex frequency $\omega=$ $\omega_{R}-i \omega_{I}$. Time evolution methods cannot determine very accurately which overtone is dominating the response, though experience has shown that the fundamental mode seems to be more excited than all others, and by definition decays much slower [1]. Most importantly, the scattering of wavepackets shows that weakly-damped modes are excited in physically interesting situations. Although not the direct focus of this work, our numerical results show no sign of a power-law tail at late stages, confirming earlier predictions [16, 35].

\section{B. Numerical Results}
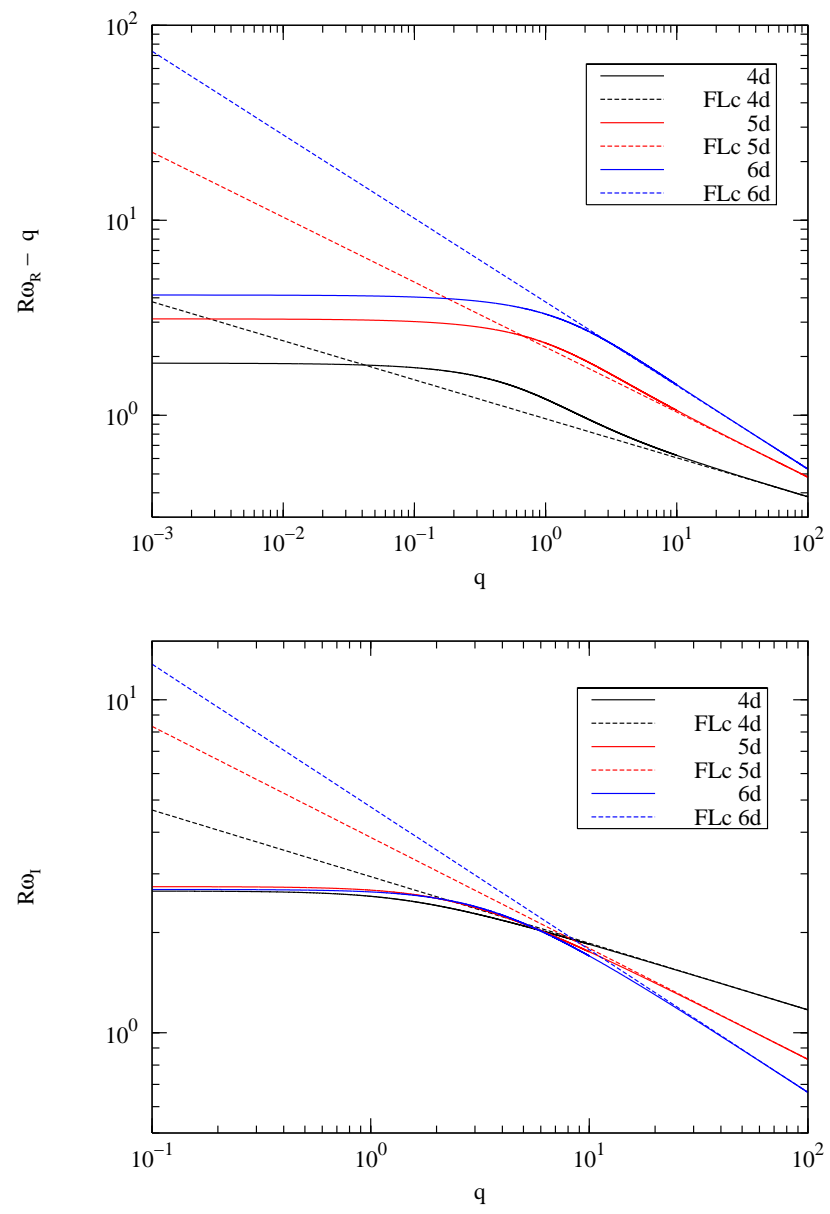

FIG. 2: Numerical results for the fundamental scalar-field (tensortype) QNM frequencies of a $r_{+} / R=1$ black brane. Upper panel: real component $\omega_{R}$ (top to bottom are $d=6,5,4$ ). Lower panel: imaginary component $\omega_{I}$ (top to bottom for $q>10$ corresponds to $d=4,5,6$ ). Dotted lines are the analytical prediction 2.77, corrected by a prefactor $a$ shown in Table

In Figure 2 we show numerical results for scalar-field (tensor-type gravitational) perturbations of a $r_{+} / R=1$ black 
brane. Similar results hold for large black holes. Low- $q$ results for $d=4,5$ are well known in the literature (See Ref. [1] and references therein), while the higher-dimensional cases are discussed in details in Ref. [36]: both $\omega_{R}$ and $\omega_{I}$ are almost independent on $q$ (or $l$ for large black holes) in this regime. For wavenumbers $q \gg r_{+} / R$, the qualitative behavior changes: $\omega_{R}$ grows linearly while $\omega_{I}$ decreases with $q$. Furthermore, it is also clear from Fig. 2 that both the subleading term in $\omega_{R}$ and the leading term in $\omega_{I}$ scale as a power of $q$ (or $l$ ). This power can be directly read from the slope of the curves of Figure 2 at large $q$. To investigate this further, we parameterize the numerical results by

$$
R \omega_{R}=q+\alpha_{R} q^{-\beta_{R}}, \quad R \omega_{I}=\alpha_{I} q^{-\beta_{I}},
$$

and we extract $\beta_{R, I}$ by least-squares. We obtain the values listed in Table \ where we also show the prediction by FL, i.e., $\beta_{R, I}=(d-3) /(d+1)$.

TABLE I: Best fit to exponents $\beta_{R, I}$, and FL's prediction, $\frac{d-3}{d+1}$.

\begin{tabular}{|c|c|c|c|c|c|c|c|c|c|c|}
\hline \multirow[t]{2}{*}{ Type } & \multirow[t]{2}{*}{$n$} & \multicolumn{3}{|c|}{$4 d$} & \multicolumn{3}{|c|}{$5 d$} & \multicolumn{3}{|c|}{$6 d$} \\
\hline & & $\beta_{R}$ & $\beta_{I}$ & FL & $\beta_{R}$ & $\beta_{I}$ & FL & $\beta_{R}$ & $\beta_{I}$ & FL \\
\hline \multirow{5}{*}{ tensor } & 0 & 0.20 & 0.20 & 0.20 & 0.33 & 0.33 & 0.33 & 0.43 & 0.43 & 0.43 \\
\hline & 1 & 0.21 & 0.20 & 0.20 & 0.34 & 0.33 & 0.33 & 0.43 & 0.43 & 0.43 \\
\hline & 2 & 0.21 & 0.20 & 0.20 & 0.34 & 0.33 & 0.33 & 0.43 & 0.43 & 0.43 \\
\hline & 3 & 0.22 & 0.19 & 0.20 & 0.34 & 0.33 & 0.33 & 0.43 & 0.42 & 0.43 \\
\hline & 4 & 0.24 & 0.20 & 0.20 & 0.34 & 0.32 & 0.33 & 0.43 & 0.41 & 0.43 \\
\hline \multirow{3}{*}{ vector } & 0 & 0.19 & 0.20 & 0.20 & 0.33 & 0.34 & 0.33 & 0.42 & 0.44 & 0.43 \\
\hline & 1 & 0.20 & 0.20 & 0.20 & 0.33 & 0.33 & 0.33 & 0.43 & 0.43 & 0.43 \\
\hline & 2 & 0.20 & 0.20 & 0.20 & - & - & 0.33 & - & - & 0.43 \\
\hline \multirow{3}{*}{ scalar } & 0 & 0.17 & 0.21 & 0.20 & 0.30 & 0.31 & 0.33 & 0.39 & 0.41 & 0.43 \\
\hline & 1 & 0.16 & 0.22 & 0.20 & 0.31 & 0.35 & 0.33 & - & - & 0.43 \\
\hline & 2 & 0.19 & 0.21 & 0.20 & 0.31 & 0.34 & 0.33 & - & - & 0.43 \\
\hline
\end{tabular}

Our numerical results are consistent with a $q^{-(d-3) /(d+1)}$ dependence of the characteristic frequencies, not only for the dimensions shown in Table \, but also for $d=7,8$ and 9. Furthermore, we computed the same modes for a $r_{+} / R=100$ black hole, and to numerical accuracy we get the same results after a rescaling by $\left(\frac{r_{+}}{R}\right)^{\frac{2 d-2}{d+1}}$ is performed. Thus, our results are also highly consistent with the functional dependence on $r_{+}, q$ as given by equation 2.7. This agreement is nicely illustrated in Fig. 2. In a log-log plot, the analytical result predicts a line with slope $-\frac{d-3}{d+1}$, which overlaps very well with the numerical results for large $q$.

We now assume the power-law behavior 2.7) in $q$ and $r_{+}$, and fit the numerical results to the following function

$R \omega^{\mathrm{Num}}=1+\left(a_{R} \operatorname{Re}\left[\Pi_{n}\right]+i a_{I} \operatorname{Im}\left[\Pi_{n}\right]\right)\left(\frac{r_{+}}{R}\right)^{\frac{2 d-2}{d+1}} q^{-\frac{d-3}{d+1}}$

thereby testing the prefactor in 2.7). If Eq.2.7 captures correctly all of the features of these modes, then $a_{R} \approx a_{I} \approx 1$. Table II summarizes our main results, with the correction factors to 2.7 for each dimension number $d$ and gravitational sector. The results in Table $\amalg$ are strong indicators that Eq.2.7 does not account for the correct quantitative behavior of these weakly-damped modes. The results are

TABLE II: Correction factors to the analytical formula (2.7).

\begin{tabular}{cccccccc}
\hline Type & $n$ & \multicolumn{2}{c}{$4 d$} & \multicolumn{2}{c}{$5 d$} & \multicolumn{2}{c}{$6 d$} \\
& & $a_{R}$ & $a_{I}$ & $a_{R}$ & $a_{I}$ & $a_{R}$ & $a_{I}$ \\
\hline \hline & & & & & & & \\
& 0 & 1.83 & 1.82 & 3.00 & 2.99 & 4.56 & 4.55 \\
& 1 & 1.86 & 1.85 & 3.12 & 3.10 & 4.82 & 4.80 \\
tensor & 2 & 1.87 & 1.86 & 3.15 & 3.13 & 4.86 & 4.90 \\
& 3 & 1.88 & 1.86 & 3.17 & 3.14 & 4.94 & 4.89 \\
& 4 & 1.86 & 1.85 & 3.18 & 3.14 & 4.96 & 4.89 \\
\hline & & & & & & & \\
& 0 & 1.00 & 1.02 & 1.80 & 1.82 & 2.92 & 2.95 \\
vector & 1 & 1.38 & 1.38 & 2.34 & 2.35 & 3.68 & 3.69 \\
& 2 & 1.53 & 1.53 & - & - & - & - \\
\hline & & & & & & & \\
& 0 & 0.29 & 0.30 & 0.77 & 0.80 & 1.59 & 1.64 \\
scalar & 1 & 0.92 & 0.93 & 1.59 & 1.65 & - & - \\
& 2 & 1.20 & 1.20 & 2.02 & 2.04 & - & - \\
\hline
\end{tabular}

consistent with real, overtone-independent, but dimensiondependent correction factors for scalar-field (tensor-type gravitational) perturbations. This correction factor grows with $d$ and might become dominant at large $d$.

Equation 2.7 is not supposed to hold for vector-type and scalar-type gravitational perturbations, but we find it captures the essential qualitative behavior with $r_{+}, q$. It can describe quantitatively the numerical results if multiplied by a real constant, which depends on the overtone $n$ and the spacetime dimension $d$. This clearly suggests a new form for $\Pi_{n}$.

\section{CONCLUSIONS AND OUTLOOK}

Our numerical results lend strong support to FL's prediction for the existence of long-lived modes in the eikonal limit. Furthermore, the functional dependence of these modes on the horizon radius and momentum $q$ is consistent with FL, but we show that the correct quantitative behavior is not. In particular, if we correct their prediction by some (real) correction factors, listed in Table II one can account extremely well for the numerical results. Taken together, our results suggest that large- $q$ tensor-type (or scalar-field) quasinormal frequencies of black holes and black branes are described by

$$
R \omega=q+a \Pi_{n}\left(\frac{r_{+}}{R}\right)^{\frac{2 d-2}{d+1}} q^{-\frac{d-3}{d+1}},
$$

where the value of $a$ depends on $d$, but it is independent on the overtone number $n$.

We also provide correction factors that would make prediction 2.7 describe well other types of gravitational quasinormal modes (vector and scalar). For such perturbations, the real constant $a$ depends not only on $d$ but also on $n$, suggesting a completely different form to $\Pi_{n}$. In any case, there is a simple and universal dependence on $r_{+}$and $q, l$ in this 
eikonal regime. Perhaps a simple interpretation in terms of geodesics can be given, as is done in asymptotically flat spacetimes [1, 37]. Clearly, more analytical and numerical studies are necessary to have a clear picture of the eikonal, weaklydamped regime of quasinormal modes of large black holes and black branes. A particularly interesting direction is to assess the degree to which these modes can be excited, which is tantamount to computing the residue of the Green function at the QNM pole. This is an important research topic in asymptotically flat spacetime, where it allows to predict how astrophysical black holes respond to external sources [38], and has also recently started to be explored in the gauge/gravity duality scenario [39, 40].

Note added in proof: We have recently been informed [41] of a mistake in one integral in FL, which introduces the cor- rection factor $a=(1 / 2)(d-1)^{(2 d-2) /(d+1)}$ in Eq. 4.1. This correction factor is consistent with all our numerical results for scalar fields or tensor-type gravitational perturbations.

\section{Acknowledgements}

We would like to thank Guido Festuccia for helpful correspondence. This work is partially supported by Fundação para a Ciência e Tecnologia (FCT) - Portugal through project PTDC/FIS/64175/2006 and by Conselho Nacional de Desenvolvimento Científico e Tecnológico of Brazil (CNPq). JM thanks Fundação Universidade Federal do ABC (UFABC) for a grant.
[1] E. Berti, V. Cardoso and A. O. Starinets, arXiv:0905.2975 [grqc].

[2] V. Ferrari and L. Gualtieri, Gen. Rel. Grav. 40, 945 (2008) [arXiv:0709.0657 [gr-qc]].

[3] K. D. Kokkotas and B. G. Schmidt, Living Rev. Rel. 2, 2 (1999) [arXiv:gr-qc/9909058].

[4] H. P. Nollert, Class. Quant. Grav. 16, R159 (1999).

[5] J. M. Maldacena, Adv. Theor. Math. Phys. 2, 231 (1998) [Int. J. Theor. Phys. 38, 1113 (1999)] [arXiv:hep-th/9711200|.

[6] E. Witten, Adv. Theor. Math. Phys. 2, 253 (1998) [arXiv:hep-th/9802150].

[7] S. S. Gubser, I. R. Klebanov and A. M. Polyakov, Phys. Lett. B 428, 105 (1998) |arXiv:hep-th/9802109].

[8] D. T. Son and A. O. Starinets, Ann. Rev. Nucl. Part. Sci. 57, 95 (2007) [arXiv:0704.0240 [hep-th]].

[9] C. Hoyos-Badajoz, K. Landsteiner and S. Montero, JHEP 0704, 031 (2007) [arXiv:hep-th/0612169].

[10] R. C. Myers, A. O. Starinets and R. M. Thomson, JHEP 0711, 091 (2007) [arXiv:0706.0162 [hep-th]].

[11] R. C. Myers and A. Sinha, JHEP 0806, 052 (2008) [arXiv:0804.2168 [hep-th]].

[12] I. Amado, M. Kaminski and K. Landsteiner, JHEP 0905, 021 (2009) arXiv:0903.2209 [hep-th]].

[13] M. Cubrovic, J. Zaanen and K. Schalm, arXiv:0904.1993 [hepth].

[14] G. Festuccia and H. Liu, arXiv:0811.1033 [gr-qc].

[15] E. Berti, V. Cardoso and P. Pani, arXiv:0903.5311 [gr-qc].

[16] G. T. Horowitz and V. E. Hubeny, Phys. Rev. D 62, 024027 (2000) |arXiv:hep-th/9909056].

[17] V. Cardoso and J. P. S. Lemos, Phys. Rev. D 64, 084017 (2001) [arXiv:gr-qc/0105103].

[18] V. Cardoso, R. Konoplya and J. P. S. Lemos, Phys. Rev. D 68, 044024 (2003) [arXiv:gr-qc/0305037].

[19] B. Wang, C. Molina and E. Abdalla, Phys. Rev. D 63, 084001 (2001) |arXiv:hep-th/0005143|.

[20] B. Wang, C. Y. Lin and C. Molina, Phys. Rev. D 70, 064025 (2004) |arXiv:hep-th/0407024].

[21] J. S. F. Chan and R. B. Mann, Phys. Rev. D 59, 064025 (1999).

[22] J. P. S. Lemos, Class. Quant. Grav. 12, 1081 (1995)
arXiv:gr-qc/9407024.

[23] C. G. Huang and C. B. Liang, Phys. Lett. A 201, 27 (1995).

[24] J. P. S. Lemos, Phys. Lett. B 353, 46 (1995) arXiv:gr-qc/9404041].

[25] R. G. Cai and Y. Z. Zhang, Phys. Rev. D 54, 4891 (1996) arXiv:gr-qc/9609065.

[26] A. S. Miranda and V. T. Zanchin, Phys. Rev. D 73, 064034 (2006) |arXiv:gr-qc/0510066].

[27] A. S. Miranda and V. T. Zanchin, Int. J. Mod. Phys. D 16, 421 (2007).

[28] H. Kodama and A. Ishibashi, Prog. Theor. Phys. 110, 701 (2003) arXiv:hep-th/0305147].

[29] A. Ishibashi and H. Kodama, Prog. Theor. Phys. 110, 901 (2003) arXiv:hep-th/0305185].

[30] A. Nunez and A. O. Starinets, Phys. Rev. D 67, 124013 (2003) arXiv:hep-th/0302026].

[31] P. K. Kovtun and A. O. Starinets, Phys. Rev. D 72, 086009 (2005) arXiv:hep-th/0506184].

[32] A. S. Miranda, J. Morgan and V. T. Zanchin, JHEP 0811, 030 (2008) arXiv:0809.0297[hep-th]].

[33] J. J. Friess, S. S. Gubser, G. Michalogiorgakis and S. S. Pufu, JHEP 0704, 080 (2007) arXiv:hep-th/0611005].

[34] G. Michalogiorgakis and S. S. Pufu, JHEP 0702, 023 (2007) arXiv:hep-th/0612065].

[35] E. S. C. Ching, P. T. Leung, W. M. Suen and K. Young, Phys. Rev. D 52, 2118 (1995) |arXiv:gr-qc/9507035.

[36] J. Morgan, V. Cardoso, A. S. Miranda, C. Molina and V. T. Zanchin, In preparation.

[37] V. Cardoso, A. S. Miranda, E. Berti, H. Witek and V. T. Zanchin, Phys. Rev. D 79, 064016 (2009) arXiv:0812.1806 [hep-th]].

[38] E. Berti and V. Cardoso, Phys. Rev. D 74, 104020 (2006) arXiv:gr-qc/0605118].

[39] I. Amado, C. Hoyos-Badajoz, K. Landsteiner and S. Montero, JHEP 07, 133 (2008) [arXiv:0805.2570 [hep-th]].

[40] I. Amado, C. Hoyos-Badajoz, K. Landsteiner and S. Montero,

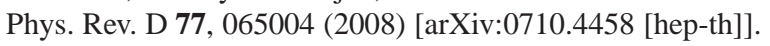

[41] G. Festuccia, private communication. 